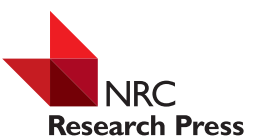

\section{Editor-in-Chief Announcement}

I am pleased to announce that Dr. Sally Pehrsson has accepted the position of Co-Editor-in-Chief for Canadian Journal of Earth Sciences. She will join Dr. Brendan Murphy in this role. Sally is a research scientist with the Geological Survey of Canada (GSC) and an Adjunct Professor at the Department of Geological Sciences, University of Saskatchewan. Her research focuses on Precambrian tectonics and global metallogeny. She has published nearly 200 peer-reviewed articles, invited reviews, maps, and reports; edited special issues of journals and bulletins; and led major projects / program development for GSC's Targeted Geoscience Initiative and Geoscience for Energy and Minerals programs during thirty years of research into the Canadian Shield.

\section{James J. Germida}

Executive Editor-in-Chief

\section{Annonce d'une nouvelle rédactrice en chef}

Je suis heureux d'annoncer que Mme Sally Pehrsson a accepté le poste de corédactrice en chef de la Revue canadienne des sciences de la Terre. Elle assumera ce rôle aux côtés de M. Brendan Murphy. Sally est chercheuse à la Commission géologique du Canada (CGC) et professeure associée au Département des sciences géologiques de l'Université de la Saskatchewan. Ses travaux portent sur la tectonique du Précambrien et la métallogénie globale. Elle a publié près de 200 articles évalués par les pairs, articles de synthèse sollicités, cartes et rapports, dirigé la publication de bulletins et de numéros spéciaux dans des revues et piloté l'élaboration de programmes et de projets importants pour les programmes Initiative géoscientifique ciblée et Géocartographie de l'énergie et des minéraux de la CGC durant trente années de recherches menées dans le Bouclier canadien.

\section{James J. Germida}

Directeur de la publication 